\section{BMJ Open} Ophthalmology

\title{
Comparative cohorts of retinopathy of prematurity outcomes of differing oxygen saturation: real-world outcomes
}

May May Choo (D) ,1,2 John Grigg, ${ }^{3}$ Elizabeth H Barnes, ${ }^{4}$ Nurliza Khaliddin, ${ }^{5}$ Tengku Ain Kamalden, ${ }^{6}$ Azanna Ahmad Kamar, ${ }^{7}$ Yao Mun Choo, ${ }^{7}$ Chin Theam Lim, ${ }^{7}$ Frank Joseph Martin ${ }^{8}$

To cite: Choo MM, Grigg J, Barnes EH, et al. Comparative cohorts of retinopathy of prematurity outcomes of differing oxygen saturation: real-world outcomes. BMJ Open Ophthalmology 2021;6:e000626. doi:10.1136/ bmjophth-2020-000626

Received 17 September 2020 Revised 27 January 2021 Accepted 28 January 2021

\section{Check for updates}

C) Author(s) (or their employer(s)) 2021. Re-use permitted under CC BY-NC. No commercial re-use. See rights and permissions. Published by BMJ.

For numbered affiliations see end of article.

Correspondence to Professor May May Choo; mmchoo@um.edu.my

\section{ABSTRACT}

Objective An ongoing third epidemic of retinopathy of prematurity (ROP) is contributed largely by developing nations. We describe a cohort of infants in a single neonatal unit where two limits of oxygen saturation were administered, to show real-world outcomes from trend in neonatology for higher oxygen to improve survival.

Methods and analysis This retrospective, comparative study of prospectively collected data in an ROP screening programme included infants indicated by gestational age $\leq 32$ weeks, birth weight $<1501 \mathrm{~g}$, ventilation for 7 days or requiring oxygen $>1$ month, who underwent dilated fundoscopic examination from age 4 weeks, every 2 weeks until full retinal vascularisation. Infants with ROP were examined weekly and treated where indicated. Data were divided into two epochs. Epoch 1 oxygen saturation targets were [88-92\%], epoch 2 targets [90-95\% (99\%)] with allowance of increase to $20 \%$ for several hours after procedures. Outcome measures included development of ROP, treatment, mortality, sepsis and intraventricular haemorrhage.

Results A total of 651 infants underwent examination between 2003 and 2016. The incidence of ROP in epoch 1 was $29.1 \%$ and epoch 2 was $29.3 \%(p=0.24)$. ROP progression doubled in epoch 2 (5 vs $11 \%, p=0.006)$, proportion of cases treated halved ( $14 \%$ vs $6 \%, p=0.0005)$, sepsis was halved $(78.5 \%$ vs $41.2 \%, p<0.0001)$ and intraventricular haemorrhage doubled $(20.2 \%$ vs $43.8 \%$, $\mathrm{p}=0.0001$ ) in epoch 2. Mortality was $4 \%$ and $0 \%$ in epochs 1 and 2, respectively.

Conclusion Incidence of ROP did not differ, although ROP cases that worsened doubled with higher oxygen targets. ROP cases requiring treatment decreased, as did sepsis and mortality. Intraventricular haemorrhage cases doubled.

\section{INTRODUCTION}

High oxygen exposure was a probable cause of retrolental fibroplasia, ${ }^{12}$ first observed by Campbell and Patz. Retrolental fibroplasia is the end-point of retinopathy of prematurity (ROP), the leading cause of blindness in premature infants. Higher oxygen is important for infant survival as shown by a 2017 Cochrane review, ${ }^{3}$ which found that oxygen saturation range of
$90 \%-95 \%$ was safer than $85 \%-89 \%$, with the lower oxygen level increasing average risk of mortality by 28 per 1000 infants treated. Many published randomised controlled trial studies initiated by paediatricians reported that higher oxygen saturation improved survival rates and reduced complications. ${ }^{4-6}$ The SUPPORT trial reported that mortality was more frequent in the lower oxygen $(85 \%-89 \%)$ group $(19.9 \%$ vs $16.2 \%, \mathrm{p}=0.04)$ although severe ROP was much less $(8.6 \%$ vs $18 \%, \mathrm{p}<0.001)$. The BOOST II trial ${ }^{4}$ reported higher mortality rate $(23.1 \%$ vs $15.9 \%$, $\mathrm{p}=0.002$ ) and higher risk of necrotising enterocolitis in infants kept at lower oxygen saturation $(85 \%-89 \%)$. The risk for ROP was reduced $(10.6 \%$ vs $13.5 \%, \mathrm{p}=0.045)$ while on lower oxygen. ${ }^{4}$ There is no study on outcomes of ROP development from the perspective of the ophthalmologist managing the disease in the real world where a change of oxygen saturation levels occurred following these publications. The current third epidemic of $\mathrm{ROP}^{7}$ is a worrying trend for developing nations. Blencowe et al stressed that $65 \%$ children who are visually impaired from ROP were from middle-income regions.

Each neonatal centre may have different saturation levelsin use and the need togenerate its own data on ROP outcomes. University of Malaya Medical Centre is a tertiary referral hospital and since 1999 maintains a screening programme for ROP in accordance with international standards. They maintained two differing saturation levels within the last 16 years. In the first epoch, oxygen saturation was kept at $88 \%-92 \%$, but in 2010 , the levels were set at $90 \%-95 \%$, with allowance of up to $99 \%$ for several hours following procedures. This study aimed to observe the trend in ROP during a 14-year period (2003-2016) where an institutional change in oxygen limits was implemented. It aimed to detect difference 


\section{Key messages}

\section{What is already known about this subject?}

- What is already known since STOP-ROP was higher oxygen at up to 99\% did not benefit cases that had developed plus disease, nor did it worsen retinopathy of prematurity (ROP). And where it did benefit those in pre-threshold stage, it brought longer dependence on oxygen and prolonged hospitalisation. SUPPORT, BOOST II and COT trials reported a limit of $95 \%$ as maximum saturation and showed improved survival, but in the real world, oxygen saturation may be higher especially after sessions of physiotherapy; the oxygen is dialled up for a few hours. BO0ST I had also delivered up to $98 \%$ saturation targets for their cohort. The reports of higher birthweight infants getting severe ROP have been observed in recent years and within ophthalmic circles, and this observation is concerning.

\section{What are the new findings?}

$>$ Our study outcome presents findings from the perspective of the ophthalmologist who has to deal with the changes from this paradigm shift. Any change in oxygen comes with much worry that we will be back to facing more severe cases of ROP. This cohort of infants from Malaysia presents a real-world situation in a less developed country in which screening for ROP is implemented for infants who are of a higher cut-off (ie, $1500 \mathrm{~g}$ as the criteria) which differed from previously reported randomised trials which used $1250 \mathrm{~g}$ as a cut-off. There is as yet no ideal treatment for this potentially blinding disease although the advent of anti-VEGF therapy has offered some salvage for disease stages previously not responsive to standard therapy using laser ablation of immature retina when ROP is present. Both therapies have its limitations.

$\checkmark$ Laser damages the retina and its aim was to preserve pre-existing developed retinal tissue whereas anti-VEGF injections carry with it risks of infection, worsening traction retinal detachment and potential for stoppage of further development of retina. Hence, after anti-VEGF therapy, risk for ROP recurring is high when an area of non-vascularisation is present, thus requiring regular screening beyond those treated with conventional laser therapy. Anti-VEGF medication has been shown to escape from the ocular barriers and may hinder tissue development elsewhere. Anti-VEGF treatment is all off-label in children. The long-term complications of use are still not reported.

- The new findings from our cohort was the observation of higher percentage of cases who developed intraventricular haemorrhages in the cohort with higher oxygen targets, contrary to what has been reported in SUPPORT and BOOST that reported improvements. Intraventricular haemorrhage is a risk factor for ROP in our previous report on extremely low birth weight infants. The COT trial also found higher oxygen was not protective of this, but their findings, as with BO0ST trials, incorporated older infants. Hence, our perspective is important to note.

\section{How might these results change the focus of research or clinical practice?}

Our findings will bring forth focus to ophthalmologists to be acutely aware of this trend towards higher oxygen delivery, and the focus of research should be more to analyse zone 1 disease or aggressive posterior ROP incidences as more neonatal units adopt a higher oxygen profile. As for clinical practice, as ophthalmologists we may anticipate higher incidence of ROP that requires more time to do screening for as well as anticipate potentially poorer visual outcome in cases with higher grades of intraventricular haemorrhage especially in grade IV which are known to cause optic nerve compromise. in ROP incidence based on these different oxygen limits and any improvement in outcomes.

\section{MATERIALS AND METHODS}

A retrospective study of data retrieved from the database of infants who underwent examination for ROP screening from 2003 to 2016 was conducted. All premature infants who were born at 32 weeks or below, birth weight $1500 \mathrm{~g}$ or less, or those requiring oxygen for at least 1 month or ventilation for 7 days were referred for ROP screening. The first examination was carried out at chronological age 4 weeks for most cases, but some allowance was made to defer by a week if an infant was deemed unfit for the examination by the neonatologist. The cohorts were divided into two groups: epoch 1 and epoch 2. Throughout epoch 1 (2003-2009), oxygen saturation was kept strictly between $88 \%$ and $92 \%$. In epoch 2 (2010-2016), oxygen was administered to maintain saturation over a higher range 90\%-95\%. Following physiotherapy or resuscitation, the oxygen saturation was increased $10 \%-20 \%$ above the baseline for several hours, which meant that saturation reached $99 \%$ and beyond before being dialled down. Frequency of examination was 2 weekly until full retinal maturation. However, in the patients with ROP, their examination frequency varied between weekly examination to 3-day reviews if their ROP stage had progressed. Progression was defined as a worsening stage or presence of plus disease if not previously present. The stages of ROP were recorded according to ICROP classification and APROP (aggressive posterior retinopathy of prematurity) is defined as an aggressive form of the disease whereby progress to a higher stage occurs rapidly.

The main outcome measures were development of any ROP, the worst stage of ROP achieved in any eye and the cases that required treatment. Treatment indications were based on ETROP $^{9}$ study indications. Two experienced paediatric neonatologists (MMC, NK) performed all the examinations and treatment throughout this period. The hospital is a tertiary referral centre, and patients admitted to the neonatal intensive care unit (NICU) remained in the hospital until weight gain of over $2.2 \mathrm{~kg}$ was achieved. All patients included in this analysis were those who qualified for ROP screening and completed their final examinations or died during the period of screening examinations. There were no changes in both epochs with respect to indications for screening and indications for treatment.

Data were analysed using SAS V.9.4 (SAS Institute, Cary, $\mathrm{NC})$. The study was conducted with institutional ethical board approval and adhered to tenets of the Declaration of Helsinki. It was not appropriate or possible to involve patients or the public in the design, or conduct, or reporting, or dissemination plans of our research. Comparisons between epochs and other predictors of outcomes were carried out with $\chi^{2}$ tests and binary logistic regression. Two-sample t-test was used for comparisons of continuous variables. Multiple logistics regression analysis 
Table 1 Characteristics of the two epochs

\begin{tabular}{|c|c|c|c|}
\hline Cohorts & $\begin{array}{l}\text { Epoch } 1 \text { (2003-2009) } \\
\text { Lower oxygen limit (88-92\%) }\end{array}$ & $\begin{array}{l}\text { Epoch } 2 \text { (2010-2016) } \\
\text { Higher oxygen limit (90-95\% (99\%)) }\end{array}$ & $P$ value \\
\hline Number (n) & 307 & 344 & \\
\hline Gestation (SD) (weeks) & $29.1(2)$ & $29.3(2)$ & 0.24 \\
\hline Birth weight (SD) (g) & $1116(309)$ & $1167(338)$ & 0.05 \\
\hline Mortality (\%) & $13 / 294(4)$ & $0 / 344(0)$ & 0.0001 \\
\hline Sepsis (including NEC) & 237/302 (78\%) & $131 / 318(41 \%)$ & $<0.0001$ \\
\hline Intraventricular haemorrhage & $61 / 302(20 \%)$ & $140 / 320(44 \%)$ & 0.0001 \\
\hline ROP cases detected & $94 / 307(31 \%)$ & $94 / 320(29 \%)$ & 0.73 \\
\hline ROP progression & 15/307 (5\%) & $34 / 314(11 \%)$ & 0.006 \\
\hline ROP cases requiring treatment & $44 / 307(14 \%)$ & $19 / 320(6 \%)$ & 0.0005 \\
\hline APROP cases & 3/304 (1\%) & $3 / 317(1 \%)$ & 0.96 \\
\hline Surfactant administered & $139 / 301(46 \%)$ & $186 / 319(58 \%)$ & 0.0025 \\
\hline Steroid administered & $244 / 302(81 \%)$ & $234 / 314(75 \%)$ & 0.06 \\
\hline Maternal PE & $72 / 302(24 \%)$ & $77 / 315(24 \%)$ & 0.86 \\
\hline \multicolumn{4}{|l|}{ IPPV } \\
\hline $\begin{array}{l}\text { Not ventilated } \\
<7 \text { days } \\
7 \text { days or more }\end{array}$ & $\begin{array}{l}84 / 307(27 \%) \\
98 / 307(32 \%) \\
125 / 307(41 \%)\end{array}$ & $\begin{array}{l}138 / 344(40 \%) \\
124 / 344(36 \%) \\
82 / 344(24 \%)\end{array}$ & $<0.0001$ \\
\hline Transfusion & 178/302 (59\%) & 139/321 (43\%) & $<0.0001$ \\
\hline Heart disease & 158/302 (52\%) & $121 / 320(40 \%)$ & 0.0003 \\
\hline
\end{tabular}

APROP, aggressive posterior retinopathy of prematurity; IPPV, invasive positive pressure ventilation; NEC, necrotising enterocolitis; PE, preeclampsia; ROP, retinopathy of prematurity.

was performed where it was necessary with confounders included in the model. Potential confounders were identified from any significantly different postnatal factors between epochs in our cohort(table 1), as well as from the initial univariable model of individual risk factors. The risk factors that were documented were present at the time of first ROP examination. Data may be obtained from a third party and are not publically available.

\section{RESULTS}

A total of 651 infants underwent ROP screening in this cohort. The infants were divided into two epochs (epoch 1 and epoch 2). The characteristics of each epoch is shown in table 1 . Gestational age at birth and birth weight were comparable (two-sample t-test was used for

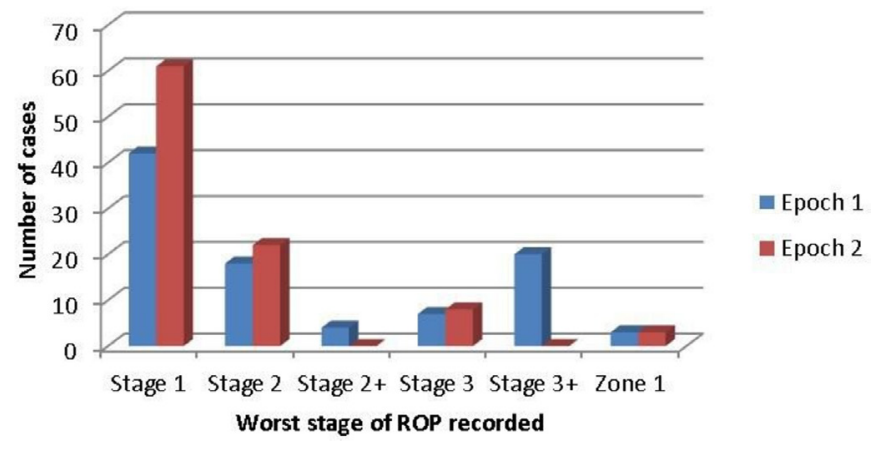

Figure 1 Bar chart of the stage of retinopathy of prematurity $(\mathrm{ROP})$ in the two epochs. comparisons of continuous variables). The incidence of ROP did not differ between the two epochs. Wider range of oxygen administered in epoch 2 was associated with more progression of ROP $(\mathrm{p}=0.006)$, but the cases that eventually required treatment was halved (14.3\% vs $5.9 \%, \mathrm{p}=0.0005$ ) in epoch 2 . Reduction of mortality and sepsis were the two main differences seen. Sepsis cases were halved $(78.5 \%$ vs $41.2 \%, \mathrm{p}<0.0001)$ in epoch 2 and mortality recorded during the screening period improved $(4.2 \%$ vs $0 \%, \mathrm{p}=0.0001)$ (table 1$)$. However, the number of intracranial bleed cases doubled in epoch 2.

Other perinatal factors compared were surfactant, administration of corticosteroids, presence of maternal pre-eclampsia, invasive ventilation, transfusion and presence of heart disease in the neonates. Surfactant was administered in a higher proportion of cases in epoch 2, which may have contributed to fewer cases who required ventilation for over 7 days. There was no evidence of a difference in corticosteroid use or maternal preeclampsia between epochs. Multiple logistic regression analysis of risk of intraventricular haemorrhage revealed an OR of $6.8(\mathrm{p}<0.0001)$ for those in epoch 2 to develop this compared with epoch 1.

Figure 1 shows the stage of ROP for the cohort. There was a marked increase in stage 1 and 2 ROP cases in epoch 2; however, stage 3+ were observed a lot more in epoch 1. Zone 1 ROP was seen in both epochs equally. The numbers of cases that progressed in epoch 1 was 

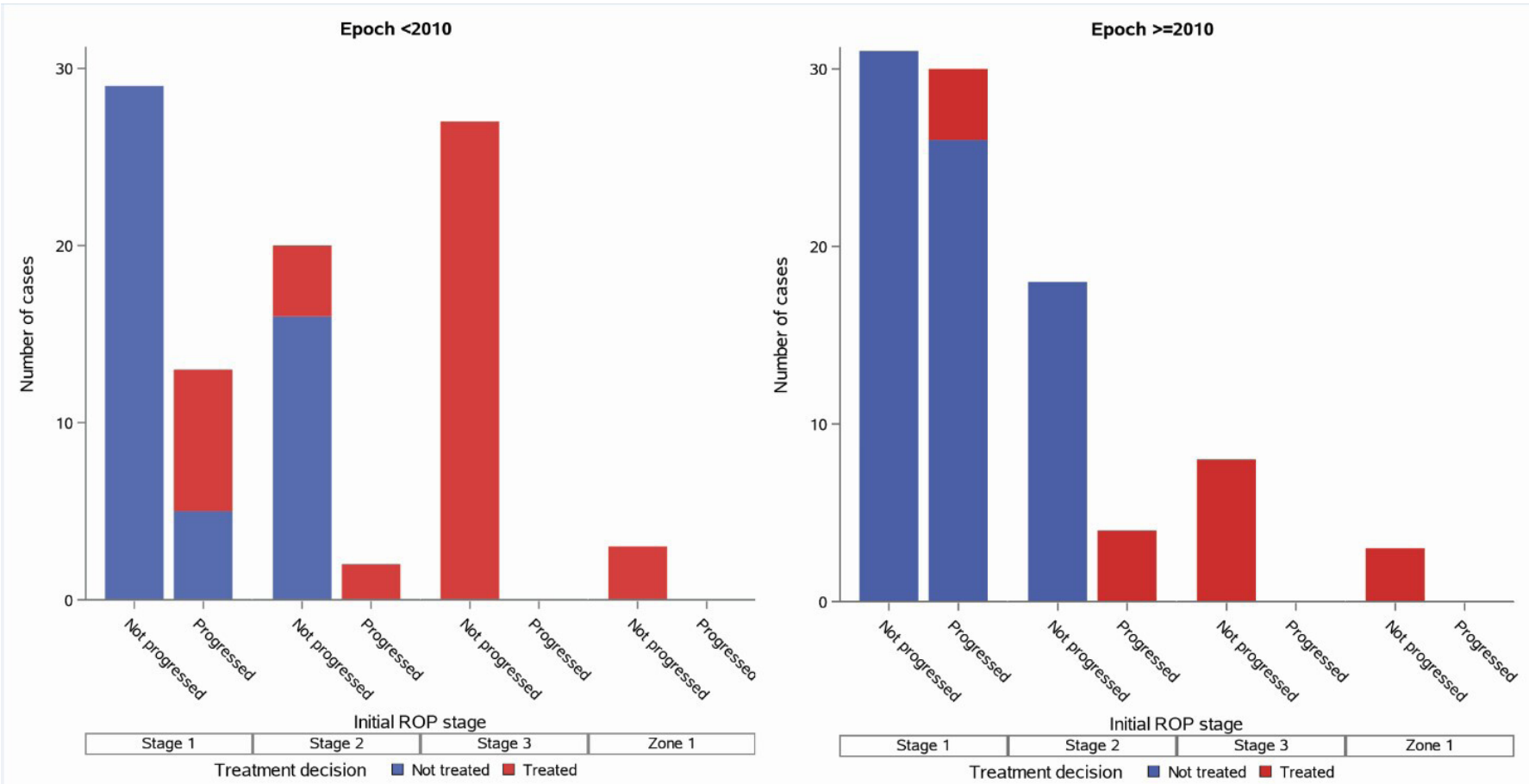

Figure 2 Numbers of cases that progressed from their initial retinopathy of prematurity (ROP) stage for both epochs.

$15 / 307(5 \%)$ versus $34 / 314(11 \%)$ in epoch 2 (table 1), excluding any cases that had been found to fulfil treatment criteria at first examination. There is apparent mismatch of ROP outcomes in this study as shown in table 1; both epochs recorded occurrence of any ROP that was comparable $(31 \%$ vs $29 \%)$, but ROP progression was observed in a higher number in epoch 2. Figure 2 shows that the initial ROP stage were mainly stages 1 and 2 in epoch 2 , whereas for epoch 1 , many cases were first detected to fulfil criteria for treatment at initial ROP examination. Progression of ROP was present if the initial ROP had worsened at a later examination to a higher stage. The indications for treatment in both epochs were stage 2 cases with plus in zone 2 disease, any stage with plus in zone 1 and stage 3 without plus disease in zone 1 . As for proportion of ROP requiring treatment, there seemed to be less in epoch 2 than epoch 1 because the total number of cases in the denominator was different between epoch 1 and epoch 2. Further analysis was carried out to ascertain if epoch 2 with higher oxygen saturation targets was protective against ROP.

Further analysis with multiple logistic regression in a combined model is shown in table 2 which revealed that higher oxygen epoch (epoch 2) was associated with OR of 2.2x for development of ROP compared with the lower oxygen epoch $(p=0.001)$, together with presence of sepsis, heart disease and ventilation of 7 days or more. Higher oxygen epoch also significantly increased odds for progression of ROP by threefold $(\mathrm{p}=0.009)$ after controlling for possible confounders. Likelihood of ROP cases requiring treatment in epoch 2 was not significantly different from epoch 1 . With ROP requiring treatment and taking into account all the possible confounders, only those receiving IPPV for 7 or more days had a significantly higher risk of ending up with treatment in epoch 2, where there was an eightfold risk (95\% CI 2.2 to 28.2, $\mathrm{p}<0.0001)$ compared with those ventilated for less than 7 days. This association should be interpreted with caution as the numbers who developed ROP requiring treatment was only 19 in epoch 2 and an even smaller required ventilation for 7 days or more. The OR for development of intraventricular haemorrhage was higher, $6.75 \mathrm{x}, \mathrm{p}<0.0001$ in the higher oxygen epoch, as were ventilation, transfusion and presence of heart disease.

Figure 2 shows the numbers of cases that progressed from their initial ROP stage for both epochs. Throughout epoch $1(<2010)$, the proportion of cases that progressed were much less. In epoch $2(\geq 2010)$, of the number of ROP cases that were initially recorded as stage 1 , many eventually progressed to a more severe stage. Furthermore, stage 2 cases that progressed to a stage requiring treatment were more in numbers in epoch 2. These contributed to the raised figure of cases which progressed in epoch 2 (10.83\% vs $4.88 \%)$.

\section{DISCUSSION}

Curtailed oxygen delivery began by 1954 when the first clinical trial showed that this reduced severity of retrolental fibroplasia. ${ }^{10}$ Retrolental fibroplasia is the endpoint of untreated severe ROP. A consensus meeting of paediatricians in 1968 agreed that only severely apnoeic infants be given supplemental oxygen and frequent eye examinations by ophthalmologists must be available. ${ }^{11}$ In 1999, STOP-ROP trial found benefits in administration of increased oxygen (96\%-99\%) saturation in ROP cases without plus disease. ${ }^{12}$ Thus, STOP-ROP paved the way 


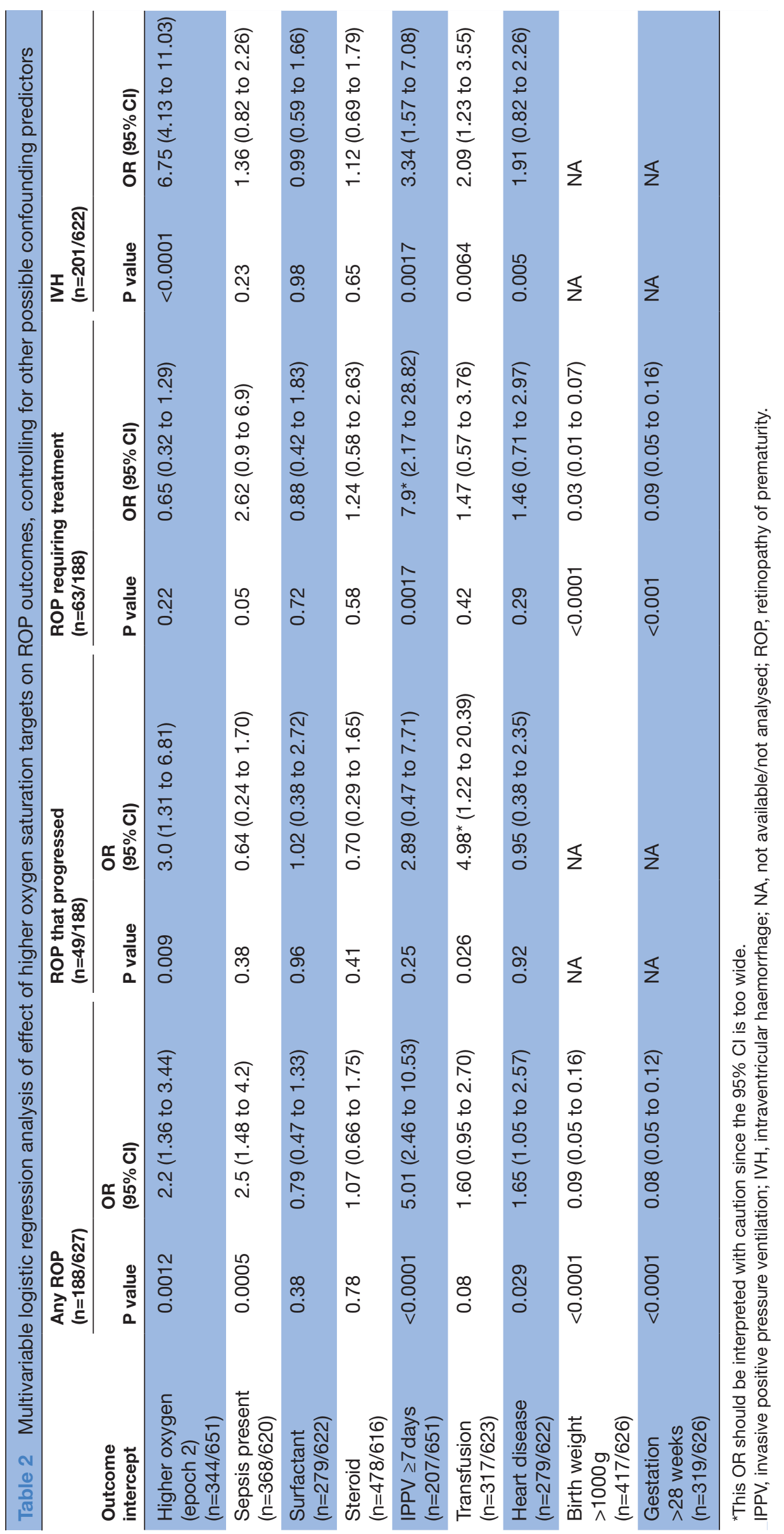


for neonatologists to re-explore increase in oxygen saturation to improve survival.

McGregor et $a l^{13}$ compared infants who had prethreshold ROP at the time of diagnosis who were on higher oxygen saturation (on room air $>94 \%$ ) versus another group receiving oxygen below $95 \%$ in HOPE-ROP trial. They found that infants exposed to $>94 \%$ oxygen saturation had less risk of progression (8). HOPE-ROP cases did better compared with STOP-ROP cases in ROP outcome. The result was $25 \%$ versus $46 \%$ progressed to threshold. These results showed that maintaining oxygen saturation above $95 \%$ was beneficial for some infants with pre-threshold ROP.

Several large paediatric trials were designed to prove that a higher oxygen saturation was needed to boost survival rates. ${ }^{4} 1415$ Table 3 shows comparisons between our oxygen study parameters with these trials. As our study was based on a pre-existing screening programme, the birth weight used to screen cases was $1500 \mathrm{~g}$ or less. Hence, our results do not reflect better outcomes since most of these clinical trials recruited infants below $1250 \mathrm{~g}$ or lower gestational age. The higher oxygen targets in Askie et al (BOOST I) ${ }^{15}$ were quite similar to our targets. Askie et al (BOOST I ${ }^{15}$ and Schmidt et al (COT) ${ }^{6}$ did not find any differences to survival rates, whereas SUPPORT and the BOOST II trials reported improved survival in their group receiving higher oxygen targets. Our results concur with SUPPORT and BOOST II trials for lowered mortality in the group maintained on higher oxygen saturation.

The incidence of ROP between epochs in our study were not significantly different, similar to findings of the other cohorts. However, SUPPORT and BOOST II showed a significant difference in ROP cases requiring treatment (severe ROP), where incidence doubled in the high oxygen arm $(8.6 \%$ vs $17.9 \%$, RR 0.52$)$ in SUPPORT. BOOST II trials reported $10.6 \%$ vs $13.5 \%$ $(\mathrm{p}=0.04)$ favouring lower oxygen group. In contrast, our cohort reported a reduction in severe cases that required treatment. This is likely due to the beneficial effects of supplemental oxygen towards some stages of ROP with plus that were seen in HOPE-ROP and STOP-ROP trials and the fact that we included larger infants in our study. Colaizy et $a l^{16}$ reported better outcome in ROP in their cohort which received supplemental oxygen to achieve oxygen saturation $>94 \%$, where cases were less likely to progress to a stage requiring treatment. Similarly, BOOST I trial ${ }^{15}$ also found no significant difference in severe ROP cases between higher oxygen (95\%-98\%) and lower oxygen (91\%-94\%). In Sarsgard's metaanalysis, both STOP-ROP ${ }^{12}$ and $\mathrm{COT}^{6}$ trials found that higher oxygen did not increase the number of cases that developed severe ROP. ${ }^{17}$

Analysis of ROP cases which showed progression from initial diagnosis has not been reported. Progression of ROP was defined as changing to a higher stage or progressing to a stage with plus from the first ROP stage detected. We observed from our cohort a higher percentage of ROP cases that progressed (worsened) during epoch 2 compared with epoch 1, $10.83 \%$ versus $4.88 \%(\mathrm{p}=0.006)$. As mentioned in methodology, any cases with ROP were examined weekly; however, if there was a progression, the cases were examined more frequently. This indirectly adds to the burden for the ophthalmology team managing ROP cases.

There is no published literature on the effect of higher oxygen on modifying other risk factors for ROP which is presented in our study. Our analysis showed higher oxygen was associated with increased OR for ROP progressing to a higher stage but not all progressed to require treatment. However, for those cases that required treatment, higher oxygen was not a significant association, but the presence of postnatal risk factors like sepsis, and ventilation of 7 days or more were. The independent risk factors for ROP requiring treatment from our centre that was previously reported were birth weight $(<750 \mathrm{~g})$ and gestational age $(<28$ weeks $) .{ }^{18}$ In this current study comparing epochs of differing oxygen targets, the difference in birth weight and gestational age were not significant, hence the possible protective effect of higher oxygen targets for ROP requiring treatment within our cohort seems probable. Furthermore, when all the possible confounding risk factors were included into the final model, higher oxygen targets were twice as likely to be associated with development of ROP and had three times higher odds for progression of the disease compared with infants in the lower oxygen epoch. As mentioned earlier, HOPE-ROP and STOP-ROP found higher oxygen in infants improved some stages of ROP. To further explain on the twofold risk of developing ROP in infants in the higher oxygen epoch, this could be related to the pathogenesis of the disease as postulated by the findings in animal experiments where Ashton et al found that kittens exposed to $70 \%$ oxygen resulted in constriction of retinal capillaries. ${ }^{19}$ This would result in ischaemia and followed by production of vascular endothelial factors that promotes neovascularisation. Hence, in the early period where area of immature retinal vasculature is larger, the effect of higher oxygen saturation will result in higher risk for development of retinopathy of prematurity.

Oxygen saturation range have increased ${ }^{20-26}$ over the years. The American Association of Pediatrics guidelines for oxygen therapy in 2012 stated that $\mathrm{SpO}_{2}$ be kept between $85 \%$ and $95 \%$ in preterm infants. ${ }^{27}$ $\mathrm{WHO}^{28}$ published recommendations on keeping oxygen between $88 \%$ and $95 \%$ for infants who are born preterm $<32$ weeks' gestation. ${ }^{28}$ European guidelines increased the oxygen limits from $85 \%-93 \%$ in 2010 to $90 \%-94 \%$ in $2019 .{ }^{27} 29$

SUPPORT and BOOST II reported no difference in intraventricular haemorrhage (IVH), but our cohort showed that epoch 2 had higher number of infants with IVH. This condition is caused by ruptures of fragile veins in the immature brain and is a major problem in NICUs. ${ }^{30}$ A possible explanation could be higher oxygen 


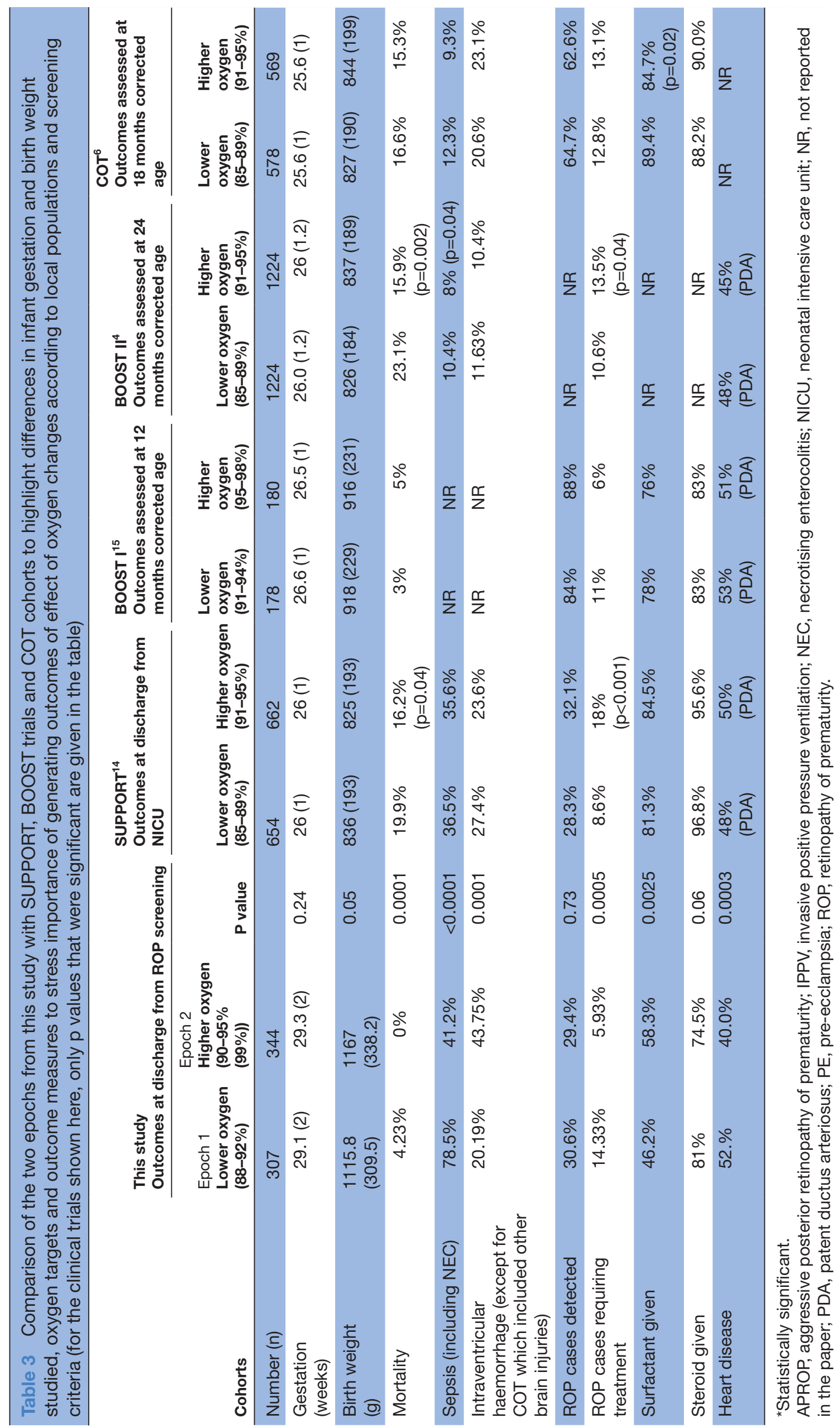


and hypocarbia from ventilation, which resulted in cerebral vasoconstriction fluctuations that have led to rupture of venules and subsequent bleed. ${ }^{31}$ Severe grade $4 \mathrm{IVH}$ is associated with hydrocephalus. ${ }^{32}$ A neurodevelopmental outcome report of the SUPPORT cohort did not show any significant difference in their cases at 2 years, ${ }^{33}$ although their cohort, like in BOOST trial, found higher oxygen protective against IVH. ${ }^{414}$ On the contrary, COT reported lower oxygen was protective against brain injury (including IVH), ${ }^{6}$ similar to our findings.

Although the trend for higher oxygen aims to improve mortality and morbidity in premature infants, Hartnett and Lane cautioned, following results of SUPPORT and BOOST, that the best oxygen profiles to reduce ROP risk while optimising preterm infant health remained unknown. ${ }^{34} \mathrm{~A}$ recent meta-analysis of these five trials by Manja $e t a l^{35}$ revealed that aside from SUPPORT, none of the other trials' initial data showed any benefit to mortality rates from the higher oxygen. BOOST II reported benefits when a composite outcome was used in their analysis combining death and neurological impairment. The deaths noted in these studies included deaths at 18 to 24 months as well. The European guidelines were revised in 2016 and recommend $\mathrm{SpO}_{2}$ target between $90 \%$ and $94 \%$ for preterm infants receiving oxygen. ${ }^{29}$ Owen et al reported that for extremely premature infants, keeping oxygen below $90 \%$ lowered the risk for ROP. ${ }^{27}$ Thus, ophthalmologists must watch with some apprehension as the level of oxygen saturation has increased steadily for the benefit of survival in premature infants.

One aspect not studied in our paper was the impact of higher oxygen on development of zone 1 ROP which has poorer outcome. ELGAN study found that the higher quartile oxygen in the first 3 days of life doubled the risk for zone 1 ROP. ${ }^{36}$ In our cohort, both epochs had the same number of zone 1 cases, but the total number of cases $(n=6)$ for our entire cohort was too small for further analysis. This could be the focus of future research. Although our study is a retrospective analysis, the data were collected prospectively as they were part of the database maintained by the author. Confounding factors may have influenced the OR, and multivariate analysis to control for various confounding risk factors was used to show the effect of higher oxygen on the outcome measures. Although the target saturation range overlapped, the wider allowance of oxygen in epoch 2, up to $99 \%$, did mean that the mean target saturations differed between both epochs (being $90 \%$ for epoch 1 and $94.5 \%$ for epoch 2). In the real world and especially in countries where there is limited resource, oxygen saturation monitoring may not be available. Such monitors should be kept for each infant while they are in the NICU.

This study provides real-world results of the consequence and implications to the ophthalmologist. The higher oxygen saturation epoch gave improved survival $(\mathrm{p}=0.0001)$ and reduced sepsis $(\mathrm{p}<0.0001)$ but did not change the incidence of ROP. However, in adjusted analysis, epoch 2 was associated with higher odds for ROP.
Twice the cases of ROP that progressed $(p=0.006)$ and twice the number of intraventricular bleeds $(p=0.0001)$ were noted in the higher oxygen epoch. These findings will need to be anticipated by ophthalmologists, as neonatology units everywhere have or are converting to delivery of higher oxygen to their premature patients. This may translate to higher number of ROP cases for repeated screening examinations as it has been shown in our cohort in epoch 2, the number of ROP cases that worsened was significantly higher compared with epoch 1. The implication on this for the ophthalmic team would be to anticipate higher workload when planning for future health management. As mentioned in the introduction, we are now experiencing a third epidemic of this disease; middle income and developing nations will need to prepare well to deal with these new challenges.

We conducted electronic searches of the Ovid MEDLINE (1946 to 2 Aug 2019), Embase (1974 to 2 Aug 2019) and CINAHL (1982 to 2 Aug 2019) with the keywords oxygen and retinopathy of prematurity.

Author affiliations

${ }^{1}$ Ophthalmology UMERC, Universiti Malaya, Kuala Lumpur, Wilayah Persekutuan, Malaysia

${ }^{2}$ Save Sight Institute, The University of Sydney, Sydney, New South Wales, Australia ${ }^{3}$ Ophthalmology, The University of Sydney, Sydney, New South Wales, Australia ${ }^{4}$ NHMRC Clinical Trials Centre, The University of Sydney, Sydney, New South Wales, Australia

${ }^{5}$ Ophthalmology, University of Malaya, Kuala Lumpur, Wilayah Persekutuan, Malaysia

${ }^{6}$ Opthalmology, UMERC, University of Malaya Eye Research Centre, Kuala Lumpur, Federal Territory of Kuala Lumpur, Malaysia

${ }^{7}$ Paediatrics, University of Malaya Medical Centre, Kuala Lumpur, Wilayah Persekutuan, Malaysia

${ }^{8}$ Ophthalmology, The University of Sydney Save Sight Institute, Sydney, New South Wales, Australia

Acknowledgements Kids Research Institute, Westmead Children's Hospital and the partial support provided by the following research grants from University of Malaya, namely RG149-09HTM, THEQS-2009A and HIR H-20 001-00-E000056. MMC is supported by a fees offset from RTP assistance from University of Sydney.

Contributors MMC designed data collection tools and monitored data collection, wrote the statistical analysis plan, cleaned and analysed the data, and drafted and revised the paper. JG analysed the data and revised sections of the manuscript. EHB analysed the data and revised the paper. NK, AAK, YMC, TAK and CTL collected the data and helped to revise sections of the manuscript. FJM, MMC and JG initiated the collaborative project and revised the paper.

Funding The following university grants are acknowledged in support of research assistants including RG149-09HTM, THEQS-2009A and HIR H-20001-00-E000056.

Competing interests None declared.

Patient consent for publication Not required.

Provenance and peer review Not commissioned; externally peer reviewed.

Data availability statement The data is kept at the Kids Research Institute.

Open access This is an open access article distributed in accordance with the Creative Commons Attribution Non Commercial (CC BY-NC 4.0) license, which permits others to distribute, remix, adapt, build upon this work noncommercially, and license their derivative works on different terms, provided the original work is properly cited, appropriate credit is given, any changes made indicated, and the use is non-commercial. See: http://creativecommons.org/ licenses/by-nc/4.0/.

ORCID iD

May May Choo http://orcid.org/0000-0003-3801-1461 


\section{REFERENCES}

1 Patz A, Hoeck LE, de la Cruz E. Studies on the effect of high oxygen administration in retrolental fibroplasia. I. Nursery observations. Am J Ophthalmol 1952;35:1248-53.

2 Campbell K. Intensive oxygen therapy as a possible cause of retrolental fibroplasia; a clinical approach. Med J Aust 1951;2:48-50.

3 Askie LM, Darlow BA, Davis PG, et al. Effects of targeting lower versus higher arterial oxygen saturations on death or disability in preterm infants. Cochrane Database Syst Rev 2017;368:CD011190.

4 The BOOST II UK, Australia and New Zealand Collaborative Groups. Oxygen saturation and outcomes in preterm infants. NEJM 2013;368:2094-104.

5 Stenson BJ. Oxygen saturation targets for extremely preterm infants after the NeOProM trials. Neonatology 2016;109:352-8.

6 Schmidt B, Whyte RK, Asztalos EV, et al. Effects of targeting higher vs lower arterial oxygen saturations on death or disability in extremely preterm infants: a randomized clinical trial. JAMA 2013;309:2111-20.

7 Gilbert C, Malik ANJ, Nahar N, et al. Epidemiology of ROP update Africa is the new frontier. Semin Perinatol 2019;43:317-22.

8 Blencowe H, Lawn JE, Vazquez T, et al. Preterm-associated visual impairment and estimates of retinopathy of prematurity at regional and global levels for 2010. Pediatr Res 2013;74:35-49.

9 Good WV, Hardy RJ. The multicenter study of early treatment for retinopathy of prematurity (ETROP). Ophthalmology 2001;108:1013-4.

10 Robertson AF. Reflections on errors in neonatology: I. The "HandsOff" years, 1920 to 1950. J Perinatol 2003;23:48-55.

11 Silverman W. Oxygen therapy and retrolental fibroplasia. Journal of Pediatric Ophthalmology and Strabismu 1968;5:245-7.

12 STOP-ROP S-R, Multicenter Study Group. Supplemental therapeutic oxygen for prethreshold retinopathy of prematurity (STOP-ROP), a randomized, controlled trial. I: Primary outcomes. Pediatrics 2000;105:295-310.

13 McGregor ML, Bremer DL, Cole C, et al. Retinopathy of prematurity outcome in infants with prethreshold retinopathy of prematurity and oxygen saturation $>94 \%$ in room air: the high oxygen percentage in retinopathy of prematurity study. Pediatrics 2002;110:540-4.

14 SUPPORT. Target ranges of oxygen saturation in extremely preterm infants. Study Group of the Eunice Kennedy Shriver NICHD Neonatal Research Network. NEJM 2010;362:1959-69.

15 Askie LM, Henderson-Smart DJ, Irwig L, et al. Oxygen-saturation targets and outcomes in extremely preterm infants. $N$ Engl $\mathrm{J}$ Med 2003;349:959-67.

16 Colaizy TT, Longmuir S, Gertsch K, et al. Use of a supplemental oxygen protocol to suppress progression of retinopathy of prematurity. Invest Ophthalmol Vis Sci 2017;58:887-91.

17 Saugstad OD, Aune D. Optimal oxygenation of extremely low birth weight infants: a meta-analysis and systematic review of the oxygen saturation target studies. Neonatology 2014;105:55-63.
18 Choo MM, Martin FJ, Theam LC, et al. Retinopathy of prematurity in extremely low birth weight infants in Malaysia. $J$ Aapos 2009;13:446-9.

19 Ashton N, Ward B, Serpell G. Effect of oxygen on developing retinal vessels with particular reference to the problem of retrolental fibroplasia. Br J Ophthalmol 1954;38:397-430.

20 Wallace DK, Veness-Meehan KA, Miller WC. Incidence of severe retinopathy of prematurity before and after a modest reduction in target oxygen saturation levels. J Aapos 2007;11:170-4.

21 Deulofeut R, Critz A, Adams-Chapman I, et al. Avoiding hyperoxia in infants $<$ or $=1250 \mathrm{~g}$ is associated with improved short- and longterm outcomes. J Perinatol 2006;26:700-5.

22 Vanderveen DK, Mansfield TA, Eichenwald EC. Lower oxygen saturation alarm limits decrease the severity of retinopathy of prematurity. J Aapos 2006;10:445-8.

23 Wright KW, Sami D, Thompson L, et al. A physiologic reduced oxygen protocol decreases the incidence of threshold retinopathy of prematurity. Trans Am Ophthalmol Soc 2006;104:78-84.

24 Anderson CG, Benitz WE, Madan A. Retinopathy of prematurity and pulse oximetry: a national survey of recent practices. J Perinatol 2004;24:164-8.

25 Tin W, Milligan DW, Pennefather P, et al. Pulse oximetry, severe retinopathy, and outcome at one year in babies of less than 28 weeks gestation. Arch Dis Child Fetal Neonatal 2008;84:F106-10.

26 Flynn JT, Bancalari E, Snyder ES, et al. A cohort study of transcutaneous oxygen tension and the incidence and severity of retinopathy of prematurity. N Engl J Med 1992;326:1050-4.

27 Owen LS, Manley BJ, Davis PG, et al. The evolution of modern respiratory care for preterm infants. Lancet 2017;389:1649-59.

28 WHO. Sources and delivery of oxygen. oxygen therapy for children: a manual for health workers. Chapter 4.2.1(NLM classification: WS);29:29-30.

29 Sweet DG, Carnielli V, Greisen G, et al. European Consensus Guidelines on the Management of Respiratory Distress Syndrome 2019 Update. Neonatology 2019;115:432-50.

30 Ballabh P. Intraventricular hemorrhage in premature infants: mechanism of disease. Pediatr Res 2010;67:1-8.

31 Raichle ME, Plum F. Hyperventilation and cerebral blood flow. Stroke 1972;3:566-75.

32 Papile LA, Burstein J, Burstein R, et al. Incidence and evolution of subependymal and intraventricular hemorrhage: a study of infants with birth weights less than 1,500 GM. J Pediatr 1978;92:529-34.

33 Vaucher YE, Peralta-Carcelen M, Finer NN, et al. Neurodevelopmental outcomes in the early CPAP and pulse oximetry trial. N Engl J Med 2012;367:2495-504.

34 Hartnett ME, Lane RH. Effects of oxygen on the development and severity of retinopathy of prematurity. J Aapos 2013;17:229-334.

35 Manja V, Saugstad OD, Lakshminrusimha S. Oxygen saturation targets in preterm infants and outcomes at 18-24 months: a systematic review. Pediatrics 2017;139:e20161609.

36 Hauspurg AK, Allred EN, Vanderveen DK, et al. Blood gases and retinopathy of prematurity: the ELGAN study. Neonatology 2011;99:104-11. 